\title{
Local Measurement of Current Density by Magneto-Optical Current Reconstruction in Normally and Overpressure Processed Bi-2223 Tapes
}

\author{
S. Patnaik, D. M. Feldmann, A. A. Polyanskii, Y. Yuan, J. Jiang, X. Y. Cai, E. E. Hellstrom, D. C. Larbalestier, \\ and Y. Huang
}

\begin{abstract}
Magneto-optical current reconstruction has been used for detailed analysis of the local critical current density $\left(J_{c}\right)$ variation in monocore Bi-2223 tapes. We find, even in high quality tapes with bulk transport $j_{c} \sim 40 \mathrm{kA} / \mathrm{cm}^{2}(77 \mathrm{~K}, 0 \mathrm{~T})$, that there exist local regions which possess current densities of more than $200 \mathrm{kA} / \mathrm{cm}^{2}$. Overpressure processing at 148 bar significantly improved $J_{c}$ to $48 \mathrm{kA} / \mathrm{cm}^{2}$ by improving the connectivity. For the overpressure-processed sample we find that the current distribution is more uniform and that the maximum local current density at $77 \mathrm{~K}$ is increased almost to $300 \mathrm{kA} / \mathrm{cm}^{2}$.
\end{abstract}

Index Terms-Bi-2223, critical current density, magneto-optical imaging, overpressure processing.

\section{INTRODUCTION}

A T PRESENT, silver sheathed Bi-2223 is the only high $T_{c}$ superconducting material that can be fabricated in long lengths suitable for large-scale engineering applications [1]. The best available long length monocore tapes have critical current density $J_{c}(77 \mathrm{~K}, 0 \mathrm{~T}) \sim 40 \mathrm{kA} / \mathrm{cm}^{2}$, but there is increasing evidence that this is by no means the ultimate limiting value [2], [3]. For example, $J_{c}(77 \mathrm{~K}, 0 \mathrm{~T})$ as high as $70 \mathrm{kA} / \mathrm{cm}^{2}$ has been achieved for short multifilament samples [4]. Poor inter-grain connectivity is one reason advanced for the less than optimum current carrying capacity [5], but prior magneto-optical reconstructions suggest that problems with connectivity have many sources. For example, even very high- $J_{c}$ multifilament Bi-2223 tapes can contain as much as $20 \%$ porosity [6]. Such pores, cracks and other detrimental microstructural features are at least partially caused by the multi-step thermomechanical treatment needed to react and to densify the starting Bi-2212 precursor as it converts toward a largely Bi-2223 phase assemblage. One way to minimize porosity is to heat treat the tape with an overpressure $(\mathrm{OP})$, in which an inert gas is used to apply pressure during heat treatment to improve density of Bi-2223 [7]. One goal of OP processing is to achieve high $J_{c}$ tapes without the

\footnotetext{
Manuscript received August 6, 2002. This work was supported by the DOEEERE, with partial facilities support by the NSF funded MRSEC on Nanostructured Materials and Interfaces.

S. Patnaik is with the School of Physical Sciences, Jawaharlal Nehru University, New Delhi 110067, India (e-mail: spatnaik@mail.jnu.ac.in).

D. M. Feldmann, A. A. Polyanskii, Y. Yuan, J. Jiang, X. Y. Cai, E. E. Hellstrom, and D. C. Larbalestier are with the Applied Superconductivity Center, University of Wisconsin-Madison, WI 53706 USA (e-mail: spatnaik@facstaff.wisc.edu).

Y. Huang is with American Superconductor Corporation, Westborough, MA

01581 USA (e-mail: Yhuang@ amsuper.com).

Digital Object Identifier 10.1109/TASC.2003.812056
}

need for the intermediate rolling step, which is an important cause of residual cracking and poor connectivity, even though the IR step produces significant densification [6].

In this study, we used magneto-optical (MO) imaging to compare distributions of the local critical current density on scales of a few square micrometers in OP and normally processed (NP) samples. Our study indicates that overpressure processing not only increases the magnitude of maximum local $J_{c}$, but that it also improves overall connectivity, transport critical current density, and the frequency of the high current carrying regions.

\section{EXPERIMENTAL DETAILS}

This study is primarily based on two differently processed samples taken from the same monocore silver sheathed Bi-2223 tape. The tape was made by the usual oxide-powder-in-tube (OPIT) process [7], [10]. Each sample was processed with identical first heat treatment at 1 bar pressure (HT1) followed by intermediate rolling. The only difference between the samples occurred in the second heat treatment (HT2). The HT2 for the OP sample was done at $148 \mathrm{bar}$, and that for the NP sample was carried out at 1 bar pressure. The oxygen partial pressure in both cases was 0.075 bar. The details of sample preparation are published elsewhere [7]. The onset $T_{c}$ of the samples as determined by SQUID measurement was $\sim 111 \mathrm{~K}$. The self-field transport $J_{c}$ evaluated at $1 \mu \mathrm{V} / \mathrm{cm}$ at $77 \mathrm{~K}$ was measured to be $48 \mathrm{kA} / \mathrm{cm}^{2}$ for the OP processed and $39 \mathrm{kA} / \mathrm{cm}^{2}$ for the NP processed sample.

Both samples were mounted in a single plastic mount and imaged at the same time. The sample puck was ground to the half width of the tapes. The final polish was with $0.05 \mu \mathrm{m} \mathrm{Al}{ }_{2} \mathrm{O}_{3}$ powder in methanol so as to achieve the best possible flat surface to permit close proximity to the indicator film. The magnetic field maps were taken after cooling the samples in zero field to $77 \mathrm{~K}$ and then applying a magnetic field of $46 \mathrm{mT}$.

The magneto-optic imaging technique is based on the Faraday effect, that is rotation of the plane of polarization of light in the presence of a magnetic field. A Bi-doped iron garnet indicator film which exhibits the Faraday effect is placed on top of the superconducting sample. Polarized light reflected from a mirror coating applied to the underside of the indicator film carries information about the local variation of magnetic field produced by currents flowing in the sample. The angle of rotation of the polarization vector is directly proportional to the magnitude of the applied magnetic field over the range 


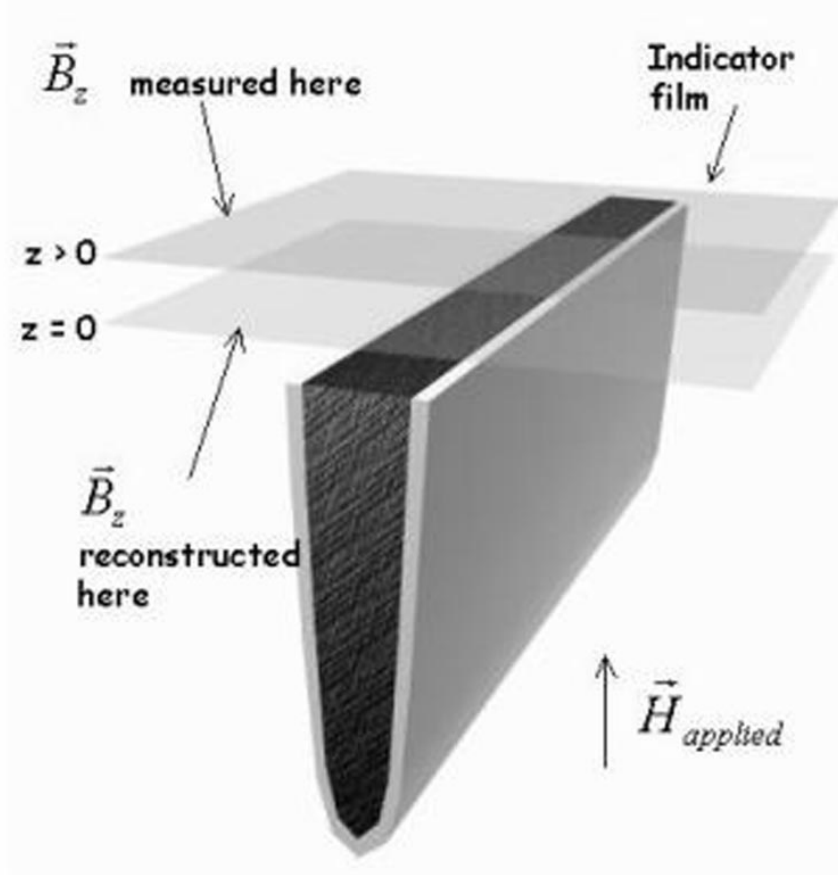

Fig. 1. The slab geometry used for the MO imaging. The specimen is half of a $2 \mathrm{~mm}$ wide tape, embedded in a polished plastic puck on which the MO indicator film is laid. The Bi-2223 thickness is $100 \mu \mathrm{m}$. The external field is applied parallel to the tape plane, that is, approximately parallel to the ab planes.

0-80 $\mathrm{mT}$ and thus produces a variation of reflected light intensity that is directly proportional to the perpendicular component of magnetic field in the imaging film.

To permit current reconstructions (MO-CR), a carefully polished, longitudinal cross section at the half width of the tape was used. This is a good approximation of a slab geometry to which the external magnetic field was applied parallel to the tape plane with $\mathbf{H} \| \mathbf{a b}$. This BSCCO tape geometry approximates two important conditions; a) 2D current flow and b) quasiinfinite sample in $z$ direction as is shown in Fig. 1. If $B_{z}(x, y)$ in the plane $z=0$ is known, then applying Maxwell's equation, the current density $\mathbf{J}$ can be derived as;

$$
\begin{aligned}
\mathbf{J} & =-\left(2 / \mu_{0}\right) \nabla \times \mathbf{B}_{\mathbf{z}} \\
|\mathbf{J}| & =\frac{2}{\mu_{0}} \sqrt{\left(\frac{\partial B_{z}}{\partial x}\right)^{2}+\left(\frac{\partial B_{z}}{\partial y}\right)^{2}} .
\end{aligned}
$$

Details of the MO-CR algorithm are described by Feldmann [8].

\section{RESULTS AND DISCUSSION}

Fig. 2 compares the MO image and the MO current reconstruction (MO-CR) for the OP and the NP samples. The sample thickness is $\sim 100 \mu \mathrm{m}$ and the reconstructions are done over a length of $\sim 500 \mu \mathrm{m}$. Both dimensions are much larger than the pixel size $(2 \times 2 \mu \mathrm{m})$ used to represent the data and the effective spatial resolution of the reconstructions which is estimated to be $\sim 5 \times 5 \mu \mathrm{m}$. Fig. 2(a) and (b) represent the actual MO image and the MO-CR for the OP sample, while Fig. 2(c) and (d) provide

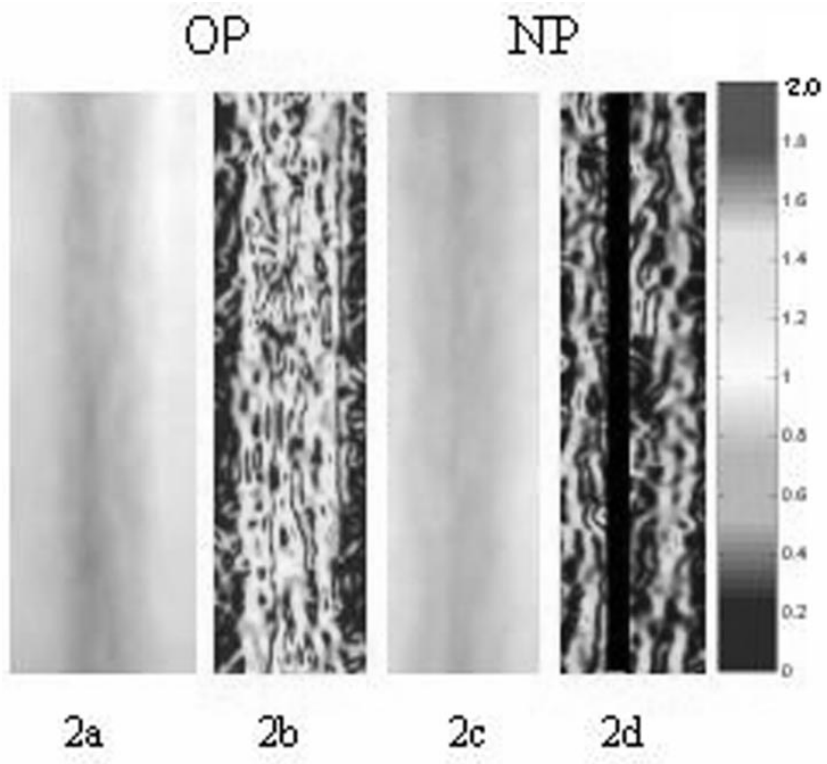

Fig. 2. (a) MO image of OP sample, (b) MO-CR of OP sample, (c) MO image of NP sample, and (d) MO-CR of NP sample. The images were taken at $T=$ $77 \mathrm{~K}$, and $\mu_{0} H=46 \mathrm{mT}$. Improvement in local $J_{c}$ for the OP sample is evident. The color scale of $J_{c}$ is linear and rises from 0 to $2 \times 10^{5} \mathrm{~A} / \mathrm{cm}^{2}$. The black bar in the NP image blanks out uncertain regions due to erratic domain structure that occurs occasionally in the ferromagnetic film.

the same information for the NP sample. The reconstructions are color coded to represent the local variability of critical current density. Dark red and dark blue represent the maximum and minimum in local current density for each plot presented.

The MO images of Fig. 2(a) and (c) represent the nonuniform nature of $B_{z}$ due to the percolative nature of current flow along the tape's polycrystalline network. This feature is even more evident in the current density maps shown in Fig. 2(b) and (d). It is striking to find that there are local regions in both samples that possess current densities up to 5 times that of the bulk transport current density. The central black line in the NP reconstruction [Fig. 2(d)] blanks out an uncertain region due to erratic domain structure that occurs occasionally in the ferromagnetic indicator film. Such domains were much less pronounced in the OP sample. As is evident from the current reconstructions, we note that the high current carrying regions are much more uniformly distributed in the OP sample. It is also remarkable that high current density regions in the OP sample are much larger in size than in the normally processed sample. This implies improved connectivity in OP sample over multiple grains, each of which is typically $\sim 20 \mu \mathrm{m}$.

Fig. 3(a) and (b) enable a direct comparison of connectivity between the OP and the NP samples at $77 \mathrm{~K}$ using two different visualizations of the MO-CR data. The color scale is always set to go from zero (dark blue) to maximum (dark red), using maxima of $X=50 \mathrm{kA} / \mathrm{cm}^{2}$ and $X=200 \mathrm{kA} / \mathrm{cm}^{2}$ in the two cases presented. Recall that the transport current densities for the OP and NP samples are $48 \mathrm{kA} / \mathrm{cm}^{2}$ and $39 \mathrm{kA} / \mathrm{cm}^{2}$ and that the field suppression of $J_{c}$ in the condition of $\mathrm{H}$ parallel to the tape plane is less than 5\%. It is evident that the high current carrying regions in the OP sample vastly outnumber those in the NP sample for both $X=50 \mathrm{kA} / \mathrm{cm}^{2}$ and $X=200 \mathrm{kA} / \mathrm{cm}^{2}$. Further, for $X=50 \mathrm{kA} / \mathrm{cm}^{2}$, we find that almost the whole OP 


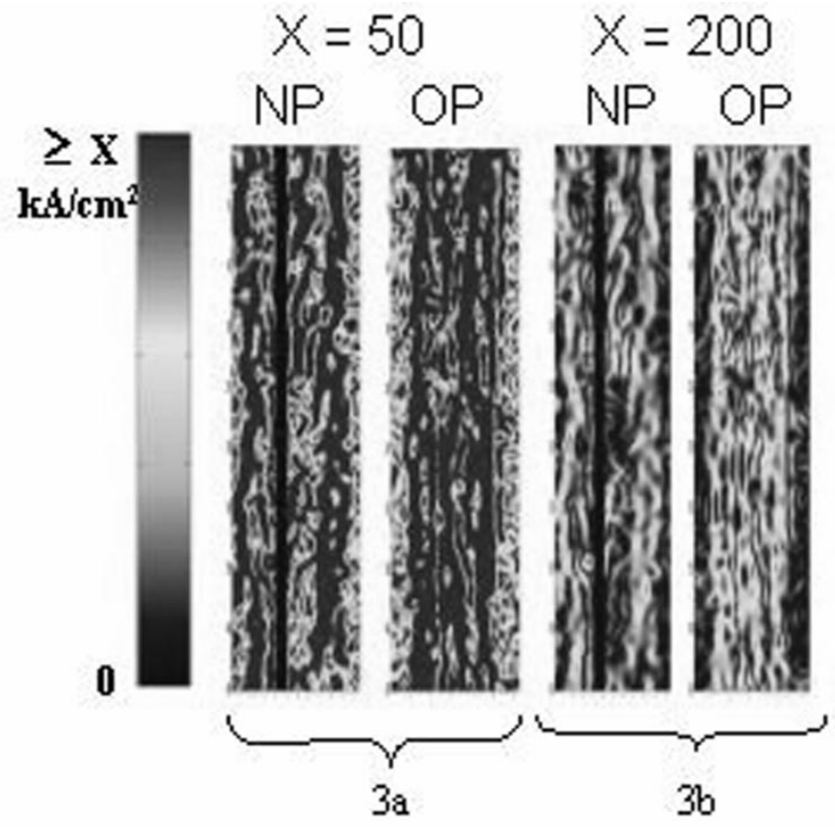

Fig. 3. Direct comparison of connectivity between OP and NP samples; (a) for upper limit value (see text) of $50 \mathrm{kA} / \mathrm{cm}^{2}$, and (b) for upper limit value of $200 \mathrm{kA} / \mathrm{cm}^{2}$. Note that there is a continuous current path for the OP sample [Fig. 3(a) right] consistent with its transport current density of $48 \mathrm{kA} / \mathrm{cm}^{2}$.

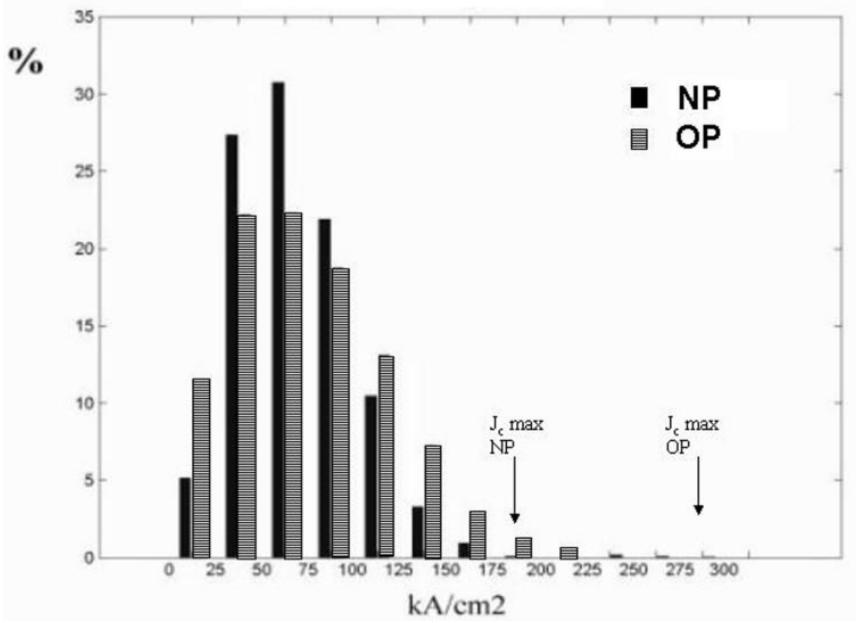

Fig. 4. Histogram of the percentage of local regions (each corresponds to $\sim 2$ $\times 2 \mu \mathrm{m}^{2}$ ) that carry a specific critical current density. The average $J_{c}$ and the local $J_{c}$ maxima are drastically improved by overpressure processing.

sample is red in color and we note that $50 \mathrm{kA} / \mathrm{cm}^{2}$ is very close to the $48 \mathrm{kA} / \mathrm{cm}^{2}$ self field transport $J_{c}(77 \mathrm{~K})$.

To obtain a quantitative estimate of the percentage of high current carrying regions, we show a histogram of the data derived from the reconstructions in Fig. 4. Each bar corresponds to a range of $J_{c}$ value between $x \mathrm{kA} / \mathrm{cm}^{2}$ and $x+25 \mathrm{kA} / \mathrm{cm}^{2}$. From the histogram, it is unambiguously evident that overpressure processing not only increases the density of local regions carrying higher $J_{c}$ as compared to the NP sample, but also improves the maximum local $J_{c}$ in the sample. While the maximum observed value for the NP sample is $\sim 200 \mathrm{kA} / \mathrm{cm}^{2}$, the OP sample possesses values of nearly $300 \mathrm{kA} / \mathrm{cm}^{2}$ at $77 \mathrm{~K}$. This

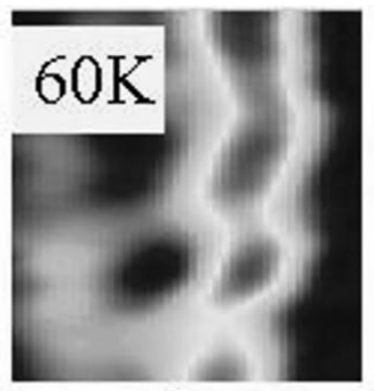

$5 \mathrm{a}$

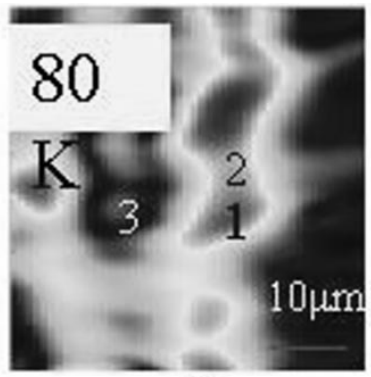

$5 b$

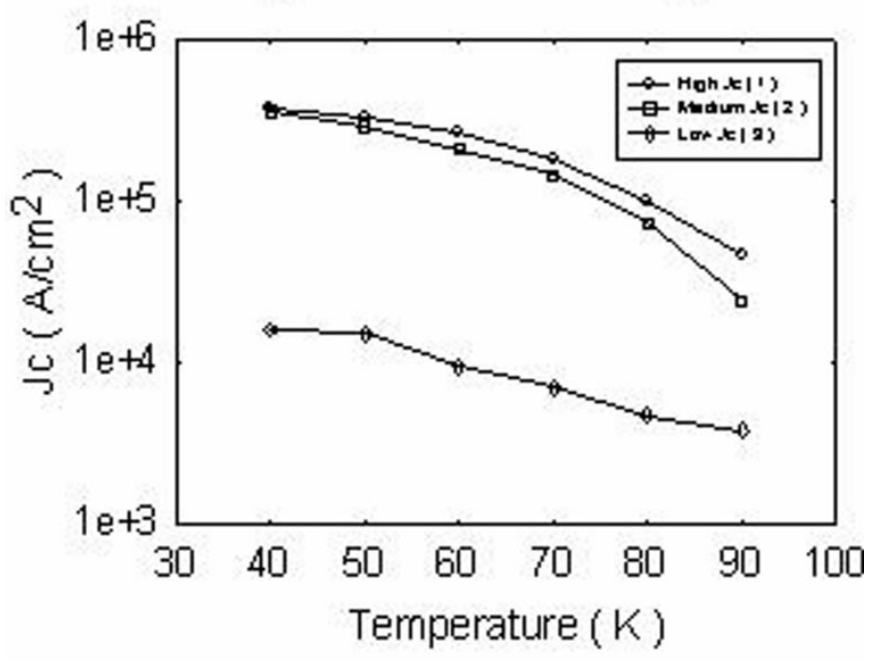

$5 c$

Fig. 5. (a) Local $J_{c}$ at $T=60 \mathrm{~K}, H=31 \mathrm{mT}$ for NP sample; (b) local $J_{c}$ at the same region at $T=80 \mathrm{~K}, H=31 \mathrm{mT}$, spot 1,2 , and 3 correspond to high, medium, and low $J_{c}$ regions, respectively; (c) temperature dependence of local $J_{c}$ at constant external field. Notice that $J_{c}$ at spot 2 does not go to 0 at $90 \mathrm{~K}$ implying the suppressed $J_{c}$ is not due to $\mathrm{Bi}$-2212 intergrowths.

is at least $30 \%$ higher than the previously reported $J_{c}$ value for the best quality Bi-2223 tapes [9]. We believe that the higher values of the OP sample represent a real improvement associated with closing up of voids, cracks and other obstacles that remain in NP processed tapes. It is not yet clear what the ultimate limit to $J_{c}$ will be for fully optimized OP processed tapes [7].

Fig. 5 presents another view of how to use MO-CR to investigate the properties of BSCCO tapes. In this case, a study of what obstructs current flow across two neighboring regions that differ in current density by about an order of magnitude is studied. Recent work has shown that 2212 phase is one such obstacle [10], [11]. To test this hypothesis, we have done MO reconstructions as a function of temperature on an NP sample. Fig. 5(a) and (b) represent reconstructions in a small region at $60 \mathrm{~K}$ and $80 \mathrm{~K}$ respectively. The high $J_{c}$ region is marked 1 , the medium $J_{c}$ region is marked 2 (the link between high current carrying regions), and the low- $J_{c}$ region is marked 3. Fig. 5(c) shows the temperature dependence of local $J_{c}$ in these 3 regions. Even at $90 \mathrm{~K}$ region 2 of medium $J_{c}$ magnitude does not drop to zero, therefore we conclude that $\mathrm{Bi}-2212$ intergrowths are not solely responsible for poor connectivity at these regions in this Bi-2223 tape. 


\section{CONCLUSIONS}

We have done extensive magneto-optical current reconstructions on $\mathrm{Bi}-2223$ tapes, which were processed under 1 bar and under 148 bar pressure. We have established that overpressure processing significantly improves the local critical current density, leading to better connected, higher $J_{c}$ regions and higher maximum $J_{c}$ values. We have observed values of $J_{c}$ of almost $300 \mathrm{kA} / \mathrm{cm}^{2}$ in the overpressure processed tape. Our study shows that connectivity is a multi parameter function and $\mathrm{Bi}-2212$ regions or intergrowths are not the sole interruption to current flow.

\section{ACKNOWLEDGMENT}

The authors wish to thank colleagues in the Wire Development Group for many useful discussions of these results.

\section{REFERENCES}

[1] D. C. Larbalestier, A. Gurevich, D. M. Feldmann, and A. A. Polyanskii, Nature, vol. 414, pp. 368-377, 2002.
[2] L. Miu, P. Wagner, U. Frey, A. Hadish, D. Miu, and H. Adrian, Phys. Rev. B, vol. 52, pp. 4553-4558, 1995.

[3] A. Attenberger, Ph.D. dissertation, Technische Universitat, Dresden, Germany, 2000

[4] A. P. Malozemoff, W. Carter, S. Fleshler, L. Fritzemeier, Q. Li, L. Masur, P. Miles, D. Parker, R. Parrella, E. Podtburg, G. N. Riley, Jr., M. Rupich, J. Scudiere, and W. Zhang, IEEE Trans. Appl. Supercond., vol. 9, pp. 2469-2473, 1999.

[5] K.-H. Müller, C. Andrikidis, J. Du, K. E. Leslie, and C. P. Foley, Phys. Rev. B, vol. 60, pp. 659-666, 1999.

[6] J. Jiang, X. Y. Cai, A. A. Polyanskii, L. A. Schwartzkopf, D. C. Larbaleastier, R. D. Parrella, Q. Li, M. W. Rupich, and G. N. Riley, Jr., Supercond. Sci. Technol., vol. 14, pp. 548-556, 2001.

[7] Y. Yuan, R. K. Williams, J. Jiang, D. C. Larbalestier, X. Y. Cai, M. O. Rikel, K. L. DeMoranville, Y. Huang, Q. Li, E. Thompson, G. N. Riley, Jr., and E. E. Hellstrom, Physica C, to be published.

[8] D. M. Feldmann, Ph.D. dissertation, Department of Physics, University of Wisconsin - Madison, USA, 2001.

[9] A. A. Polyanskii, D. M. Feldmann, S. Patnaik, J. Jiang, X. Cai, D. Larbalestier, K. DeMoranville, D. Yu, and R. Parrella, IEEE Trans. Appl. Supercond., vol. 11, pp. 3269-3273, 2001.

[10] J. Jiang, X. Y. Cai, J. G. Chandler, S. Patnaik, A. A. Polyanskii, Y. Yuan, E. E. Hellstrom, and D. C. Larbalestier, IEEE Trans. Appl. Supercond, 2002, submitted for publication.

[11] Y. B. Huang, X. Y. Cai, G. N. Riley, Jr., D. Larbalestier, D. Yu, M. Teplitsky, A. Otto, S. Fleshler, and R. D. Parrella, Adv. in Cryo. Eng., 2002 , to be published. 